\title{
An Intelligent Automated Door Surveillance System using Smart Camera
}

\author{
Rakshith M. D. \\ Department of CSE \\ SDMIT, Ujire
}

\author{
Thyagaraju G. S. \\ Department of CSE \\ SDMIT, Ujire
}

\author{
Sharath K. R. \\ Department of CSE \\ SDMIT, UJire
}

\begin{abstract}
Monitoring of areas with high security value is needed in today's security sensitive world. Such monitoring requires $24 / 7$ security for longer duration. In this paper Internet of Things (IoT) based human intrusion detection system has been proposed. This prevents unauthorized person gaining access to authorized area. The main advantage of the proposed system is to alert the organization about the intrusion along with the image that is captured using smart camera and by sending the notification to owner.
\end{abstract}

\section{General Terms}

Monitoring, Security, Organization

\section{Keywords}

Internet of Things, intrusion detection, smart camera

\section{INTRODUCTION}

In public places such as grocery stores, businesses, transportation stations, airports, and wholesale department stores automatic entrance/exit door control is widely used to eliminate the need of manually opening and closing actions. Contemporary sensor-based automatic door control technologies include infrared, ultrasonic/radio, or other wireless sensing methods. The first can be further divided into active and passive approaches. The active process emits infrared signals from the controller and captures the reflected signals to determine if there is any object close to the door. This approach is accurate and capable of identifying the position and the speed of the object but its high cost has made it less popular. The passive approach detects the infrared signals radiated by people and is the most widely used for being simple, effective, and low cost. The ultrasonic/radio approach, on the other hand, emits ultrasonic or radio waves to scan the environment and analyzes the returned signals for door access control. Surveillance means to monitor something. Due to the traditional devices and process used for securing any apartment or complexes, security in residential complexes are restricted to limited geographical locations. High Definition Surveillance Camera using raspberry pi is set up in the areas which need to be monitored. This system is motivation from other systems which explains similar systems with some disadvantages which is tried to be solved. It also includes some additional feature like data backup, push alert messages.

\section{RELATED WORK}

In this section, the author discusses about the existing door surveillance system.

Priya Patel [1], proposed "Smart surveillance and Monitoring System using Raspberry pi and Android". This system is operated via android device by owner remotely as well as locally. An IoT based android application is used for remote controlling and for sending the push notifications to the authorized person when an intrusion is detected inside the room. The proposed system provides two types of accesses: 1) Local Access: If person is connected with the local server same as the system, he can control the Raspberry pi and monitor the area in form of image/video through android application 2) Remote Access: Even if user with android device is away from the system but connected with the separate internet plan then controlling of Raspberry pi is possible with the IOT application named Dataplicity.

Adimulam Padmanabham [2], proposed "An IOT Approach for Motion Detection using ARM-Based System on Chip". This approach is based on Frame Difference Algorithm which detects the motion using Raspberry pi. The motion is captured by the camera and the difference between present frame and previous frame is calculated. Then the image is transferred to a specific folder in the Raspberry Pi. Further the image is sent from Raspberry pi to drop box automatically or command line interface. Using this technology, we can share our link to multi users allowing them to access the image whenever they want. If This Then That (IFTTT) technology is used to trigger mails automatically once the image reaches the specific folder.

A. Arun Raja [3], proposed "An Internet of Things (IOT) Based Security Alert System using Raspberry pi". This system records a video when a motion is detected and uploads it to the external server and notifies the user via text message. The remote activities can be viewed by using the proposed application and notifications can be received whenever the motion is detected.

Aamir Ansari [4], proposed "An IOT Approach for Motion Detection using Raspberry pi". This approach aims to simplify motion detection and make interfaces user friendly by sending the prompt notifications when motion is detected. A python script is used for motion detection and wput is used for storing the files on an external server.

Swapna Jadhav [5], proposed "Smart Motion Detection System Using Raspberry Pi".This system is suitable for small personal area surveillance. i.e., personal office cabin, bank locker room, parking entrance. Whenever the motion is detected through PIR sensor inside the room the image is captured through camera and temporarily stored in the raspberry pi module. Internet of Things based android application can be used remotely to view the activity and get notifications when motion is detected. The proposed Surveillance system consists of mainly two parts: 1) Hard wired surveillance system: These systems use wires to connect cameras, motion detectors, power supply and LAN cable with Raspberry pi.2) Remote Access Systems: These systems have capability to monitor and control a security system from a location away from the surveillance area through android. 
Amol Boke [6], proposed "Advanced Real Time Home Security System Based on Raspberry pi”. In this system camera will continuously capture the images and these images are compared with the reference image in the controller. If there is any difference in the captured image and the reference image, then a message will be sent to the user and at the same time the difference images are also sent. If the user is busy when the message arrives then the processor will wait some time for the feedback, if it is not received with in predefined interval then it will raise the alarm. These systems can be useful for home as well as for industrial security system. Another advantage of this system is that because of its small size and portability, it can be placed in any kind of surrounding for surveillance.

Sunil Kanzariya [7], proposed "Real Time Video Monitoring System Using Raspberry Pi". The embedded real - time video monitoring system based on ARM has been proposed in which the embedded chip and the programming techniques are employed. The central monitor which adopts Raspberry pi is the core of the whole system. Real time video transmission is widely used in surveillance, conferencing, media broadcasting and applications that include remote assistance. First, USB camera video data are collected by the embedded Linux system. All data are processed, compressed and transferred by the processing chip. Then, video data is sent to monitor the client by wireless network. This embedded monitoring system overcomes the week points of the traditional video surveillance systems, such as complex structure, poor stability, and expensive cost. It can be widely used in many fields, and also used for long distance transmission.

\section{METHODOLOGY}

The proposed system is designed by activating the code through authorized person using application on smart mobile. The application monitors any human intrusion which in turn alerts the authorized person by sending notification on smart mobile application through internet. Simultaneously the videos are captured by triggering camera through Raspberry $\mathrm{Pi}$ and then it is uploaded in cloud server so that the authorized user can monitor his authorized area from anywhere.

The proposed system also takes snapshot of the area for every 5 seconds and sends the photo to user along with the alert notification. This in turn helps the user to take necessary action against intrusion. When the owner gets alert of intrusion, as preventive measure automatic door lock and releasing gases will be done to faint the intruder. Images and videos captured by the surveillance camera will be sent to cloud server through wireless network and with help of control trigger program.

Whenever the cloud server gets data or when any changes occur, it will reflect the changes to user mobile with the help of mobile synchronization. The proposed system is designed to be operated by using two different power supply such as AC power Supply and Solar. To charge battery connected to the devices, the system enhances auto change over power sources and it also alerts the user if there is any difficulty in power sources to work.

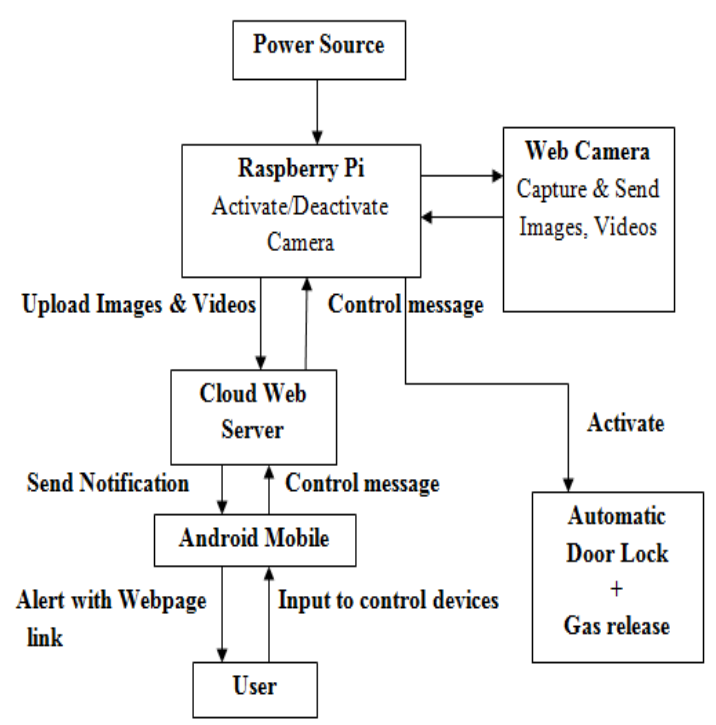

Fig 1: Architecture of the Proposed System

Images captured by the camera will be given to the Raspberry pi for processing. In processing, images are cropped and classified using edge detection and classification algorithms to find out whether the intruder is human or not. This increases the accuracy of the intrusion detection. Based on the detection result from Raspberry pi, the alert about the intrusion will be given to the authorized user's android mobile. The architecture of the proposed system is shown in the above figure.

\section{RESULTS AND DISCUSSION}

The experimental results obtained by implementing the proposed human intrusion detection system are discussed below:

\subsection{Capturing \& Storing the Image in the Database}

In this step the image of the user who wants to enter the home will be captured by using the smart camera. It will then be stored in the database where the trained images are stored.

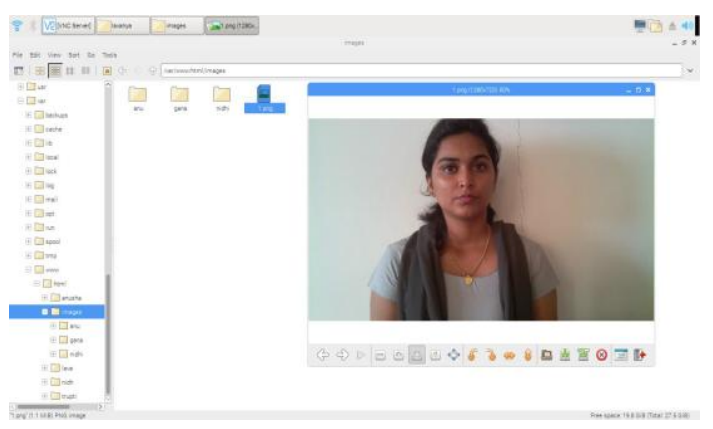

Fig 2: Captured \& stored image 


\subsection{Comparing Captured Image with the Stored Image}

In this step the captured image is compared with the training dataset i.e., stored images. If the captured image matches with the stored image then the door opens automatically. Then the notification is sent to the owner of the house.

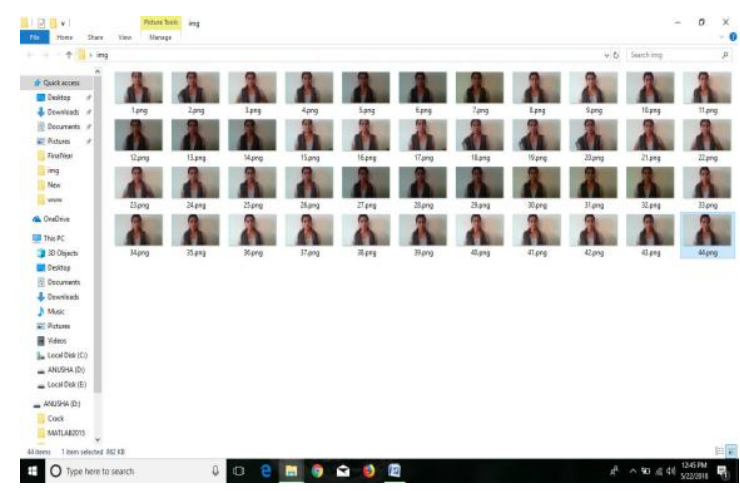

Fig 3: Training dataset

\subsection{Sending Notification to the Owner}

In this step the notification is sent to owner of the house when both authorized \& unauthorized user tries to enter the house. The below snapshot shows the notification sent to owner when authorized user tries to enter the house.

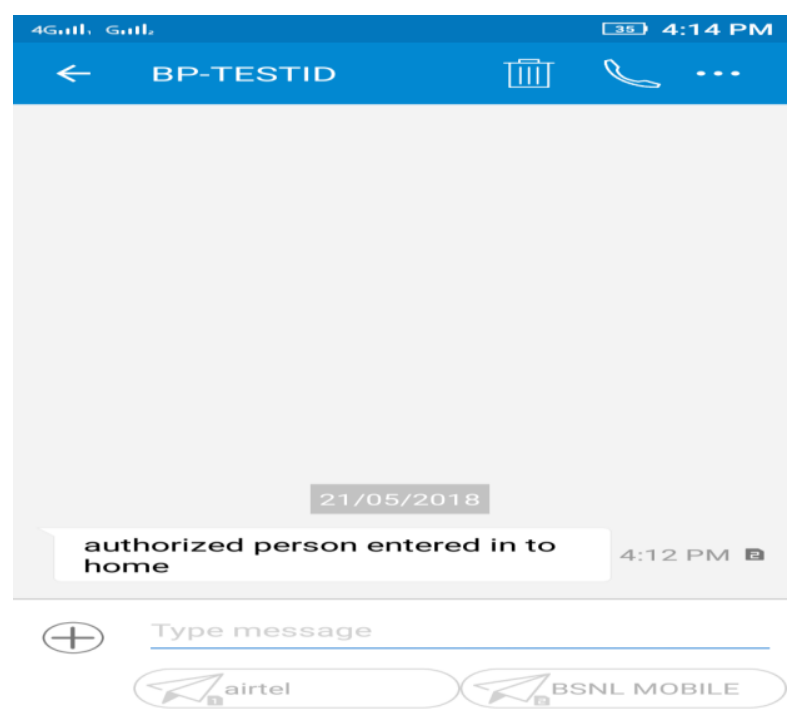

Fig 4: Sending Notification to the Owner

\section{CONCLUSION AND FUTURE SCOPE}

A smart surveillance system has been designed \& implemented to fulfil the needs of the user in surveillance area. It has countless applications and can be used in different environments and scenarios. For instance, at one scenario it can be used by any person working in industry to be aware of the activity being happened at their own working places, in his absence. While at another instance it can be used for spy purposes at bank lockers, storage houses. The advantage of the proposed system is it provides information to the user about what is happening in surveillance area by sending notification.

As a future scope an application can be developed which includes push notification as well as the controlling power of raspberry pi from the window. User can also be provided facility to view captured image remotely on this application. Live video streaming can be added as per the user requirement. Automatic power management can also be incorporated to make the system to go for sleep mode when it is no longer in active mode. The proposed system can also be extended further by adding additional infrared emitting facility to detect the people face if they wore the mask on his/her face. Apart from this we can interface sensors like Gas sensors, Smoke sensors and Fire sensors to give alerts respectively.

\section{REFERENCES}

[1] Priya B Patel, Dr. K.R. Bhatt, Viraj M Choksi and M. B. Potdar. Smart Surveillance and Monitoring System using Raspberry Pi \& Android.

[2] Adimulam Padmanabham and Venugopal, 2016. An Internet of Things Approach for Motion Detection using ARM-Based System On Chip.

[3] Arun Raja, A., Naveedhab., Niranjanadevic, G and Roobini, V. 2016. An Internet of Things (IOT) Based Security Alert System using Raspberry Pi, Asia Pacific International Journal of Engg Science.

[4] Aamir Nizam Ansari, Mohamed Sedkyl, Neelam Sharma, Anurag Tyagil.2015. An Internet of Things Approach for Motion Detection using Raspberry Pi, International Conference on Intelligent Computing and Internet of Things.

[5] Sahil, R., Amol Boke., Pragathi Kene. Advanced Realtime Home Security Based on Raspberry $\mathrm{Pi}$, International Journal of Pure \& Applied Research in Engineering \& Technology.

[6] Sunil Kanzariya., Vishal Vora. 2015. Real-time Video Monitoring using Raspberry Pi. National Conference on Emerging Trends in Computer, Electrical \& Electronics.

[7] Khushbu Mehta., Niti Gupta.2016. Vision-based Realtime Monitoring Security System for Smart Home.

[8] Serkan Akbas, Suat Ozdemir. 2014. Performance Evaluation of PIR Sensor Deployment in Critical Araea Surveillance Networks.IEEE International Conference on Distributed Computing in Sensor Systems (DCOSS), pp.327-332.

[9] Parashiva Murthy, B M., Inchara S. 2016.Motion Detection using IoT Mechanism. International Journal of Engineering Research in Computer Science \& Engineering.

[10] Sundas Zafar. 2015.Motion Detecting Camera Security with Email Notification \& Live Streaming using Raspberry Pi. 